\title{
Science Lives: Video Portraits of Great Mathematicians
}

In mathematics, beauty is a very important ingredient... The aim of a mathematician is to encapsulate as much as you possibly can in small packages - a high density of truth per unit word. And beauty is a criterion. If you've got a beautiful result, it means you've got an awful lot identified in a small compass.

- Michael Atiyah

Hearing Michael Atiyah discuss the role of beauty in mathematics is akin to reading Euclid in the original: You are going straight to the source. The quotation above is taken from a video of Atiyah made available on the Web through the Science Lives project of the Simons Foundation. Science Lives aims to build an archive of information about the main developments in mathematics in the twentieth century, recounted by some of the people responsible for those developments. Through videotaped conversations and biographical narratives, Science Lives provides engrossing personal glimpses of a vibrant field and the people who have shaped it.

The foundation hopes to create a comprehensive, lasting resource that will be widely available to students of mathematics and to the public. It is anticipated that the series will eventually be broadened to include interviews with luminaries across all the basic sciences.

So far, twenty-five videotaped conversations have been made, and as of August 2012 nine have been posted on the site. The videos were made by George Csicsery, a filmmaker who has created several acclaimed documentaries about mathematicians. His hand in making the videos is unmistakable, not only in the quality of the images and sound but also in the aesthetic choices of camera angle and background. For each video there are links to biographical materials available on the Web. In addition, some of the videos are

DOI: http://dx.doi.org/10.1090/noti914 accompanied by narrative profiles written by noted mathematics biographers.

Hugo Rossi, director of the Science Lives project, said that the first criterion for choosing a person to profile is the significance of his or her contributions in "creating new pathways in mathematics, theoretical physics, and computer science." A secondary criterion is an engaging personality. With two exceptions (Atiyah and Isadore Singer), the Science Lives videos are not interviews; rather, they are conversations between the subject of the video and a "listener", typically a close friend or colleague who is knowledgeable about the subject's impact in mathematics. The listener works together with Rossi and the person being profiled to develop a list of topics and a suggested order in which they might be discussed. "But, as is the case with all conversations, there usually is a significant amount of wandering in and out of interconnected topics, which is desirable," said Rossi.

The videos have an intimate, relaxed feeling and convey a clear sense of personality. The conversations were carried out in places of comfort to the subject, Rossi said: for John Nash, in old Fine Hall at Princeton University; for Pierre Deligne, in the library at the Institute for Advanced Study; for Yuri Manin, in his home. "We have learned from Csicsery that the environment is a very important factor in the quality of the conversation," Rossi noted.

The conversations cover topics of interest to the people being profiled, consistent with the goal of providing insight into mathematical developments of the twentieth century. The technical level of the conversations therefore varies according to the interests of the profile subjects and the topics discussed. The amount of personal material also varies according to how much the profile subjects wish to discuss their personal lives. The videos are organized into short chapter-like sections with descriptive titles so that viewers can steer to the conversational threads they will find most interesting. There are also keys that delineate general themes, such as history, philosophy, mathematics, and the mathematics profession; asterisks indicate parts that are more technical. The total time of each interview is about two hours. 


\section{Science Lives Conversations}

Videos available on the Science Lives website (https://simonsfoundation.org/mps-science1ives):

\section{Michael Atiyah}

Egbert Brieskorn (listener: Anna Pratoussevich)

Pierre Deligne (listener: Robert D. MacPherson)

Friedrich Hirzebruch (listener: Matthias Kreck)

Robert D. MacPherson (listener: Robert Bryant)

Yuri Manin (listener: David Eisenbud)

Paul J. Sally Jr. (listener: Diane Hermann)

Isadore M. Singer

Chen-Ning Yang (listener: George Sterman)

\section{... And More To Come}

Conversations with the following individuals have been filmed and are in the editing stages.

Alfred Aho (listener: Mihalis Yannakakis)

Alexandre Chorin (listener: Jamie Sethian)

William Browder (listeners: Alejandro Adem, Andrew Ranicki, and Sylvain Cappell)

John H. Conway (listener: Alex Ryba)

Michael Freedman (listener: Robion Kirby)

Phillip Griffiths (listener: Mark Green)

Mikhail Gromov (listener: Alfredo Hubard)

Richard Karp (listener: Christos Papadimitriou)

Robion Kirby (listener: Michael Freedman)

Peter Lax (listener: Robert Kohn)

Laszlo Lovasz (listener: Avi Wigderson)

Cathleen Morawetz (listeners: Marsha Berger and Margaret Wright)

John Nash (listeners: Harold Kuhn and Charles Fefferman)

Louis Nirenberg (listener: Jalal Shatah)

James Simons (listener: Jeff Cheeger)

Elias Stein (listeners: Charles Fefferman)

S.R. Srinivasa Varadhan (listener: Jalal Shatah)
By providing a vivid and personal look at some of the great recent developments in mathematics, Science Lives helps to bring human faces to a subject that is often seen as impersonal and forbidding. It is an inspiring and edifying resource for mathematicians, students, and the general public.

- Allyn Jackson

\section{About the Simons Foundation}

The Simons Foundation was established in 1994 by mathematician and investor James Simons and his wife, Marilyn. The foundation's mission is to advance the frontiers in research in mathematics and the basic sciences.

The Simons Foundation has supported many institutions and individuals in the mathematical sciences around the globe. It has helped fund the building of lecture facilities at the Mathematical Sciences Research Institute in Berkeley and at the Institut des Hautes Études Scientifiques outside Paris as well as the creation of the Simons Center for Geometry and Physics at Stony Brook University. In 2010 the foundation launched its Mathematical and Physical Sciences program, which awards grants via open application procedures and also supports the Science Lives project. The latest addition to the program is the Africa Mathematics Project, intended to bolster mathematics scholarship on the African continent. In addition to Science Lives, the foundation's website offers a series of articles and videos of interest to mathematicians, including the Simons Science Series, Simons Symposia, and the videos of George Hart.

The foundation also supports research in the life sciences and autism research.

Please visit the website simonsfoundation . org for more information. 